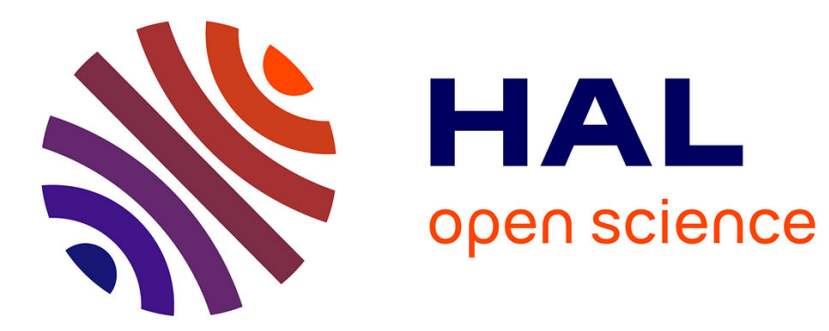

\title{
Les objectifs et les critères de sélection. Les qualités maternelles des ruminants allaitants : exemple des facilités de vêlage et de l'allaitement
}

François Ménissier, Jean Sapa, J.P. Poivey

\section{- To cite this version:}

François Ménissier, Jean Sapa, J.P. Poivey. Les objectifs et les critères de sélection. Les qualités maternelles des ruminants allaitants : exemple des facilités de vêlage et de l'allaitement. Productions Animales, 1992, 1992, pp.135-145. hal-02709010

\section{HAL Id: hal-02709010 \\ https://hal.inrae.fr/hal-02709010}

Submitted on 1 Jun 2020

HAL is a multi-disciplinary open access archive for the deposit and dissemination of scientific research documents, whether they are published or not. The documents may come from teaching and research institutions in France or abroad, or from public or private research centers.
L'archive ouverte pluridisciplinaire HAL, est destinée au dépôt et à la diffusion de documents scientifiques de niveau recherche, publiés ou non, émanant des établissements d'enseignement et de recherche français ou étrangers, des laboratoires publics ou privés. 
F. MÉNISSIER, J. SAPA et J.-P. POIVEY*.

INRA Station de génétique quantitative et appliquée 78352 Jouy-en-Josas Cedex.

* INRA Station d'amélioration génétique des animaux BP 2731326 Castanet-Tolosan Cedex
Les objectifs et les critères de sélection

\section{Les qualités maternelles des ruminants allaitants : exemple des facilités de vêlage et de l'allaitement}

Résumé. Les qualités d'élevage ou qualités maternelles mettent en cause des relations complexes entre la mère et ses produits, de leur conception à leur sevrage. L'analyse du déterminisme biologique des facilités de naissance puis de l'allaitement et de la croissance avant sevrage illustre ces implications et répercussions sur la pertinence du choix des critères et objectifs de sélection pour les qualités maternelles.

Améliorer les qualités maternelles est un des deux principaux objectifs de sélection des ruminants à viande, notamment pour maîtriser la phase de "naissage" dans les troupeaux de reproduction (Ménissier 1988). En effet, les troupeaux naisseurs se caractérisent à la fois par une efficacité économique du travail ("revenu courant") généralement basse et assez variable, et par un "revenu disponible" très sensible aux variations tant des recettes liées aux jeunes et mères de réforme commercialisés que des dépenses résultant des charges directes (alimentation complémentaire, frais vétérinaires, I.A., ...) et de structures (charges : sociales, foncières, financières, ...) (ITEB 1991). Dans ces systèmes, l'importance du capital d'exploitation (matériel, cheptel, bâtiments, stocks) est un handicap majeur puisqu'il représente pratiquement 10 ans de "revenu courant" pour un naisseur de bovins. Les fluctuations du niveau des performances techniques ont alors des répercussions notables et cumulatives sur le revenu escompté, pouvant engendrer des variations considérables de "revenu courant" (figure 1). La sélection des qualités maternelles visera donc essentiellement à accroître la part des recettes au travers de la productivité des femelles en reproduction (nombre et qualité des jeunes produits / mère entretenue) tout en réduisant les charges directes (restriction alimentaire hivernale, manipulations minimales des animaux, ...).

Les qualités maternelles impliquées correspondent à une grande diversité d'aptitudes (Ménissier 1975 et 1988) ayant trait aussi bien à la capacité de reproduction des mères (précocité, longévité, fertilité, prolificité, ...) ou à leur aptitude à élever leur progéniture (lactation, comportement, ...), qu'à leur capacité d'ingestion et d' "épargne alimentaire" ou à leur adaptation aux contraintes du milieu d'élevage (rusticité, ...). Les multiples mécanismes biologiques sousjacents au déterminisme de ces aptitudes sont importants à considérer pour préciser les critères de sélection relatifs à toutes ces qualités maternelles. L'adap-
Figure 1. Incidence de la variation marginale de revenu des principaux paramètres techniques sur le revenu final d'un élevage "naisseur" bovin. Région Limousine, 50 vaches et 50 ha de prairies, pour $122250 \mathrm{~F}$ de revenu escompté (recettes charges). Incidence cumulée : + ou $-40 \%$ du revenu escompté (source : ITEB 1991).

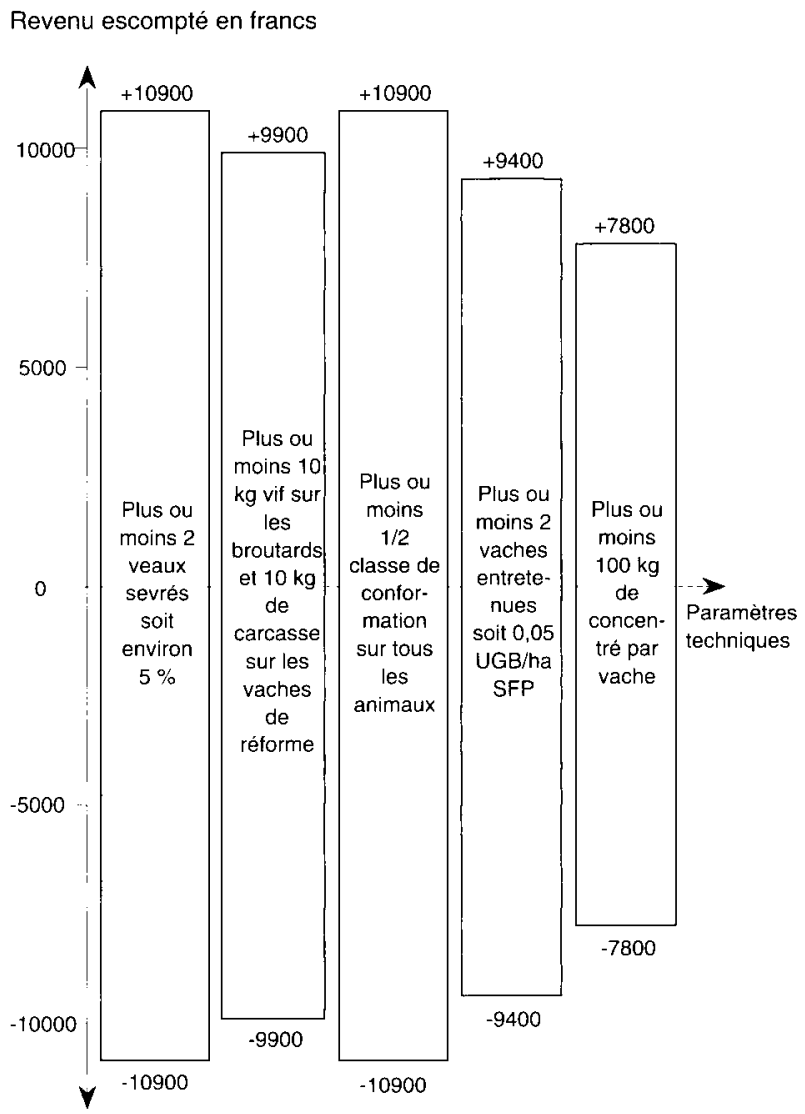


tation au milieu et la fécondité étant abordées par ailleurs, nous limiterons notre analyse d'une part aux facilités de naissance (bovins) et d'autre part à l'aptitude à l'allaitement (ovins et bovins).

\section{1 / Facilités de naissance}

\section{1 / Importance et répercussions}

La fréquence et les répercussions des difficultés de naissance des veaux sont un facteur limitant de la productivité des bovins à viande.

Ces difficultés obligent à une surveillance particulière du troupeau durant la saison de mise bas voire à des interventions pour aider à la naissance du veau ; d'où des coûts supplémentaires et des charges de travail accrues, de moins en moins compatibles avec l'agrandissement des troupeaux et l'intensification du travail de l'éleveur qui en résulte. Les risques de mortinatalité des veaux sont environ de 2 à 5 fois supérieurs lorsque leur naissance est difficile (tableau 1) en raison du traumatisme subi (anoxie, hypothermie, immunité colostrale déficiente, ...) (Ménissier et Petit 1984). De plus la fécondité des mères est considérablement perturbée, parfois aussi leur capacité d'allaitement; ce qui est incompatible avec la maîtrise de la saison de vêlage et l'augmentation de la productivité du troupeau de reproduction.

La fréquence des difficultés de naissance est non négligeable dans bon nombre de systèmes d'exploitation des races à viande, ou du moins est un frein à une plus large utilisation de ces races (Ménissier et Foulley 1978 et 1979 ). C'est le cas des races paternelles de croisement sur vaches laitières ou de races rustiques, où l'accroissement des risques de dystocies exclut le croisement industriel sur les primipares (tableau 2). En race pure, les races à viande spécialisées de grande taille et surtout les races mixtes allaitantes présentent des fréquences notables de difficultés de naissance - en particulier chez les primipares
Tableau 2. Incidence du croisement industriel avec des taureaux Charolais sur la fréquence des difficultés de naissance (\% de notes $3+4$ )

\begin{tabular}{|c|c|c|c|}
\hline \multirow{2}{*}{ Mode d'élevage } & \multicolumn{2}{|c|}{ Race laitière } & \multirow{2}{*}{$\begin{array}{c}\text { Source } \\
\text { d'information }\end{array}$} \\
\hline & P'Holstein & Normande & \\
\hline \multicolumn{4}{|l|}{ En race pure } \\
\hline ler vêlage & 8,0 & 7,3 & Contrôle laitier \\
\hline 2-12ème vêlage & 2,9 & 2,4 & Caen "I \\
\hline \multicolumn{4}{|l|}{ En croisement } \\
\hline ler vêlage & 26,8 & 17,5 & $\begin{array}{c}\text { Contrôle } \\
\text { descendance }\end{array}$ \\
\hline 4-7ème vêlage & 10,1 & 5,6 & OGER \\
\hline
\end{tabular}

(1) Manfredi et al 1990. (2) Renand 1990 - non publié.

(figure 2) - que seul des systèmes d'élevage moins extensifiés et s'interdisant un premier vêlage précoce (notamment à 2 ans) peuvent supporter.

\section{2 / Causes}

En dehors de quelques cas pathologiques ou accidentels, la plupart des difficultés résultent d'une incompatibilité morphologique foeto-maternelle, c'est à dire d'un excès de taille du foetus par rapport au canal pelvien de sa mère lors de son expulsion. Ainsi dans les situations où les difficultés de naissance sont fréquentes, près de $50 \%$ de leur variabilité est associée à celle de la taille du foetus (poids à la naissance), 10 à $30 \%$ à celle de la surface du détroit pelvien antérieur (ouverture pelvienne) de la mère, et $10 \%$ ou plus à l'état de préparation et au comportement de la mère au moment de la mise bas (figure 3 ).

En fait ces composantes agissent par "effet de seuil" sur la fréquence des dystocies (figure 4) : la fréquence ne s'accroît notablement en fonction du poids à la naissance du veau que lorsque ce poids dépasse la taille permise par l'ouverture pelvienne. Ces effets de seuil relatifs à des mères de même âge et résultant
Tableau 1. Répercussions des difficultés de naissance surla productivité des troupeaux de bovins allaitants.

Sources: ITEB 1991 -

Elevages Charolais $(F)$; Ménissier 1979 - Station Charolaise UTC d'Agonges (S).

\begin{tabular}{|c|c|c|c|c|}
\hline \multirow[t]{2}{*}{ Critère } & \multicolumn{4}{|c|}{ Mode de naissance } \\
\hline & $\begin{array}{l}\text { facile sans } \\
\text { assistance }\end{array}$ & $\begin{array}{c}\text { facile } \\
\text { avec aide }\end{array}$ & $\begin{array}{l}\text { extraction } \\
\text { forcée }\end{array}$ & par césarienne \\
\hline \multicolumn{5}{|l|}{ Mortalité des veaux $(\%)$} \\
\hline (F) $\quad 0-48$ heures & 2,6 & 3,3 & 16,6 & 5,3 \\
\hline après 48 heures & 2,2 & 2,4 & 8,2 & 5,6 \\
\hline (S) $\quad 0-48$ heures & 3,9 & 4,7 & 19,8 & 16,8 \\
\hline après 48 heures & 6,4 & 5,7 & 7,7 & 12,2 \\
\hline \multicolumn{5}{|l|}{$\begin{array}{l}\text { Taux de gestation } \\
\text { après mise bas }(\%)\end{array}$} \\
\hline (F) & 91,1 & 89,8 & 78,6 & 73,2 \\
\hline$(\mathrm{S})$ & & & 79,5 & 60,7 \\
\hline \multicolumn{5}{|l|}{ Taux de réforme $(\%)$} \\
\hline$(\mathbf{F})$ & 7,0 & 5,5 & 14,1 & 14,6 \\
\hline \multicolumn{5}{|l|}{ Production laitière $(\mathrm{kg} / \mathrm{j})$} \\
\hline (S) à 120 jours & & & & \\
\hline \multicolumn{5}{|l|}{ Croissance des veaux $(\mathrm{g} / \mathrm{j})$} \\
\hline (S) $\quad 0-120$ jours & & & & \\
\hline \multicolumn{5}{|l|}{ Poids à la naissance ( $\mathrm{kg}$ ) } \\
\hline (S) & & & & \\
\hline
\end{tabular}


Figure 2. Fréquence des difficultés de naissance selon le rang de vêlage (d'après Giraudeau et al 1990 et Manfredi 1990).

$\%$ de naissances difficiles (notes $>2$ )

$20^{-}$

15

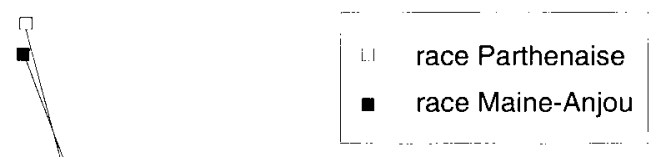

10

5

0

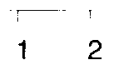

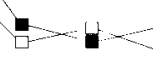

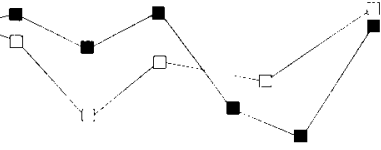

de l'interaction entre taille du veau et taille de l'ouverture pelvienne, caractérisent en quelque sorte l'aptitude au vêlage d'une race (Ménissier 1979).

\section{3 / Déterminisme génétique}

L'analyse génétique des difficultés de naissance nécessite le recours à un modèle de décomposition des effets génétiques plus élaboré (Ménissier 1979) (figure 3), prenant en compte des "effets génétiques directs" (effets du génotype du veau sur l'expression de ses performances) et des "effets génétiques maternels" (effets sur l'expression des performances du veau, résultant de l'incidence du génotype de sa mère à travers les effets de ses caractères maternels), voire des "effets génétiques foetaux" (effets résultant de l'incidence du génotype du foetus sur l'expression des caractères maternels - ce pourrait être le cas d'une partie des phénomènes de préparation à la mise bas puisque le déclenchement de la parturition est surtout sous contrôle foetal).

Les difficultés de naissance sont ainsi à considérer comme deux aptitudes selon que l'on s'adresse aux variations de facilités de naissance entre pères des veaux ("effet père") ou à celles de facilités de vêlage entre mères ("effet mère"). Dans le cas de l'effet père, les facilités de naissance correspondent à des effets génétiques directs à travers ceux relatifs notamment à la taille du veau. Au contraire, l'effet mère englobe à la fois les effets génétiques directs sur la taille du veau comme précédemment, et les effets génétiques maternels sur la croissance foetale et ceux résultant de l'incidence des caractères maternels (ouverture pelvienne, ...). Cet effet mère est analogue à un "effet grand-père maternel" lorsque l'on s'adresse aux différences entre pères des vaches. Le critère de sélection des facilités de naissance ne sera pas nécessairement le même selon que l'on veut améliorer l'un et/ou l'autre de ces effets, tout en souhaitant intervenir ou non plus particulièrement sur une des composantes.

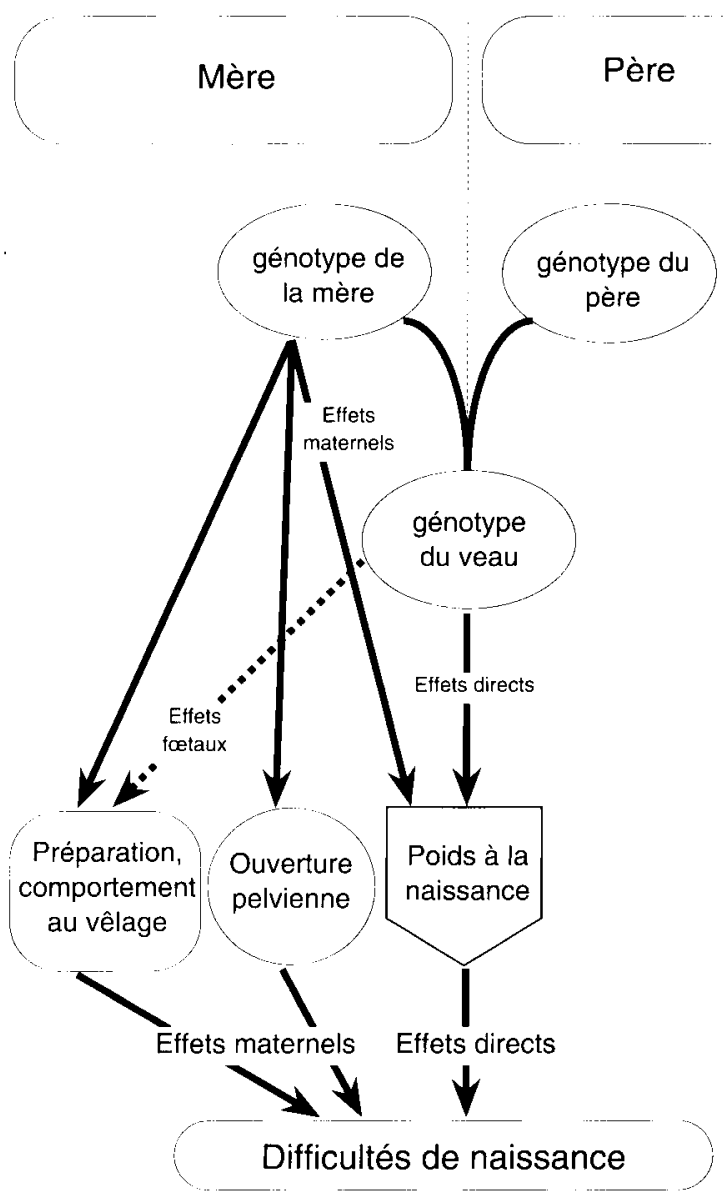

Figure 3.

Déterminisme des difficultés de naissance.
Figure 4. Effet "seuil" du poids de naissance selon l'ouverture pelvienne. (2 230 vêlages à 2 ans - Station Charolaise 1991).

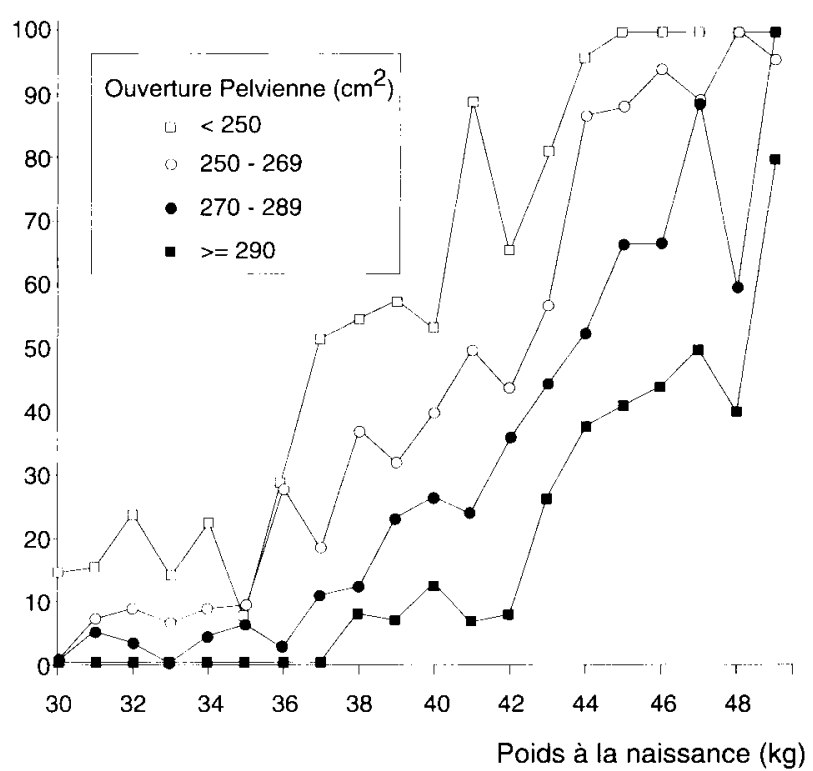


Des principaux paramètres génétiques relatifs aux difficultés de naissance des bovins à viande (tableau 3 ), ressortent plusieurs aspects dominants (Foulley et Ménissier 1978 et 1979, Ménissier et Frisch 1992).

- Les effets génétiques directs (effet père) pour les difficultés de naissance sont faiblement héritables surtout lorsque la fréquence des dystocies est faible. L'utilisation d'une statistique adaptée aux variables discrètes conduit à des héritabilités supérieures. Il n'est pas surprenant d'observer une corrélation génétique étroite entre difficultés et poids à la naissance. Ce dernier étant plus héritable, il constitue un bon prédicteur des effets génétiques directs pour les facilités de naissance. Par ailleurs la croissance foetale (poids à la naissance) étant génétiquement corrélée à la croissance post-natale, la sélection des aptitudes bouchères engendre une réponse positive sur le poids à la naissance qui, selon l'effet "seuil" des bovins exploités, aura une répercussion plus ou moins importante sur les risques de dystocie.

- En tant qu'effet mère ou grand-père maternel, les difficultés de vêlage sont également modérément héritables et leur héritabilité s'accroît avec leur fréquence (primipares vêlant précocement par exemple). Notons que la corrélation entre effet mère et effet père pour les difficultés tout comme pour le poids à la naissance, est positive mais pas très forte. De ce fait la sélection sur les seuls effets pères (ou génétiques directs) n'améliorera que faiblement les facilités de vêlage des mères (effets mères); ce qui confirme que l'effet mère est déterminé par des caractères en partie différents de l'effet père, tels que les caractères maternels (ouverture pelvienne, ...) et les effets maternels sur la croissance foetale. Cette faible liaison entre effet père et effet mère est aussi à rapprocher de la corrélation négative entre effets directs et effets maternels pour le poids à la naissance et la durée de gestation, traduisant l'existence d'une régulation foeto-maternelle de la croissance foetale. Une prédiction de l'effet mère pour les facilités de vêlage peut être obtenue en associant l'ouverture pelvienne de la mère (caractère héritable) à l'effet mère pour le poids à la naissance. La sélection de l'effet mère pour les facilités de vêlage, en mettant en jeu la taille et la précocité de développement (ou degré de maturité) du veau à la naissance (croissance foetale) et de la femelle primipare (ouverture pelvienne, format), a inévitablement des répercussions sur les caractéristiques biologiques prédominantes et inversement. Ainsi la sélection des aptitudes bouchères, par un accroissement du format, une maturité plus tardive et une muscularité plus importante qui pourraient en résulter chez les mères, risque d'engendrer une détérioration des facilités de vêlage des mères. L'importance de cet antagonisme reste à prouver ; c'est un des objectifs du programme expérimental "vachotron 2 " ("incidence d'une sélection sur la croissance musculaire") mis en oeuvre par l'INRA.

\section{4 / Contrôle et évaluation des reproducteurs}

\section{a / Critères contrôlés}

Trois types de performances peuvent être enregistrées selon la sophistication des systèmes de contrôle : la note de difficulté de naissance qui traduit l'intensité de l'assistance nécessaire à l'expulsion du nouveau-né ( $1=$ facile sans assistance, 2 =facile avec aide, $3=$ extraction forcée, $4=$ césarienne) et permet de connaître la fréquence des naissances faciles (1 et 2) ou difficiles ( 3 et 4 ) ; le poids peu après la naissance, mesuré ou parfois estimé, pour apprécier la taille du

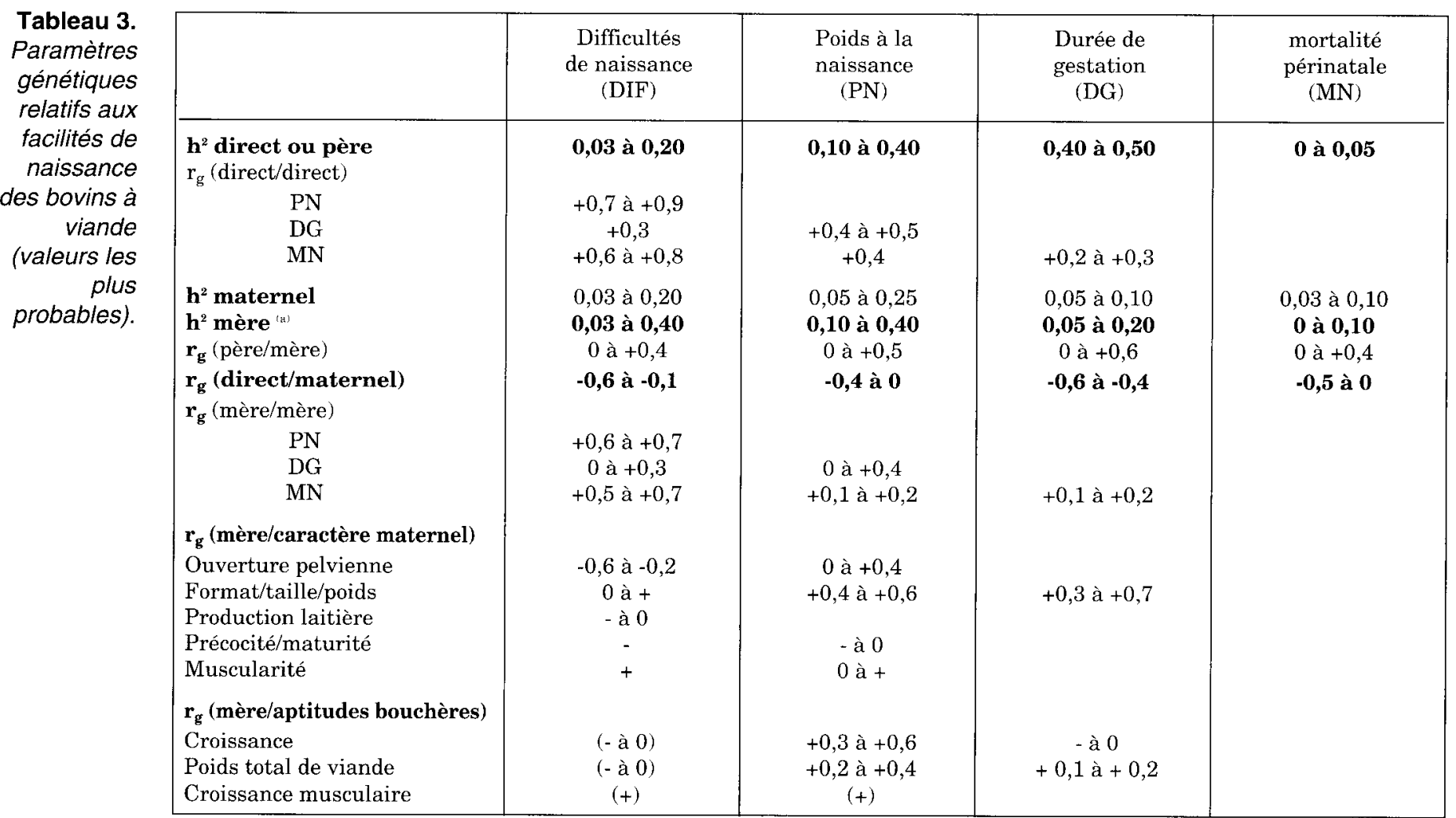

() Valeurs incertaines.

(a) ou grand-père maternel. 
veau à la naissance ; l'ouverture pelvienne (surface) et la morphologie externe mesurées en station soit après le vêlage pour les primipares soit vers l'âge de 15 mois pour les jeunes taureaux.

\section{b / Reproducteurs évalués}

L'évaluation des difficultés de naissance correspond en fait à deux aptitudes évaluées : soit l'effet père ("facilités de naissance") pour les taureaux, soit l'effet mère ("facilités de vêlage" ou "aptitude maternelle au vêlage") pour les taureaux et pour les vaches. Les conditions de naissance ou de vêlage n'étant qu'un des critères d'évaluation parmi ceux des divers programmes de sélection mis en oeuvre pour les bovins à viande, leur système d'évaluation est dépendant de celui des aptitudes bouchères ou des qualités maternelles dans lesquels ils sont insérés (Ménissier 1988) (tableau 4).

- L'évaluation des taureaux d'IA pour les aptitudes bouchères se pratique par procréation planifiée en ferme de 50 à 100 veaux croisés ou de race pure dont un échantillon sera contrôlé en station ou ateliers d'engraissement. Le poids à la naissance des veaux fourni par l'éleveur sert à estimer l'indice "facilités de naissance" (effet père) des taureaux. Une table de correspondance entre cet indice et le risque de difficultés de naissance attendu selon les caractéristiques des vaches (race, parité, région) auxquelles seront accouplés les taureaux, accompagne ces indices. L'agrément "aptitudes bouchères" des taureaux oblige à une sélection minimale sur les facilités de naissance.

- L'évaluation des taureaux d'IA pour les qualités maternelles est réalisée sur un échantillon de 20 à 25 filles de race pure procréé en ferme mais contrôlé en station afin de maximiser l'expression des différences d'effet mère pour les difficultés de vêlage (vêlage à 2 ans et à âge constant, pères des veaux identiques) tout en mesurant les composantes morphologiques des mères. L'indice "facilités de vêlage" des taureaux (effet grand-père maternel) est estimé à partir de la fréquence des vêlages difficiles et une évaluation est fournie pour chaque caractère maternel (ouverture pelvienne, poids, préparation, ...). L'indice "facilités de vêlage" est une des composantes de l'indice combiné à partir duquel se fait l'agrément "qualités maternelles" des taureaux. En outre, avec les notes et poids à la naissance des veaux procréés en ferme pour obtenir l'échantillon de filles, un indice "facilités de naissance" (effet père) est élaboré pour ces taureaux.

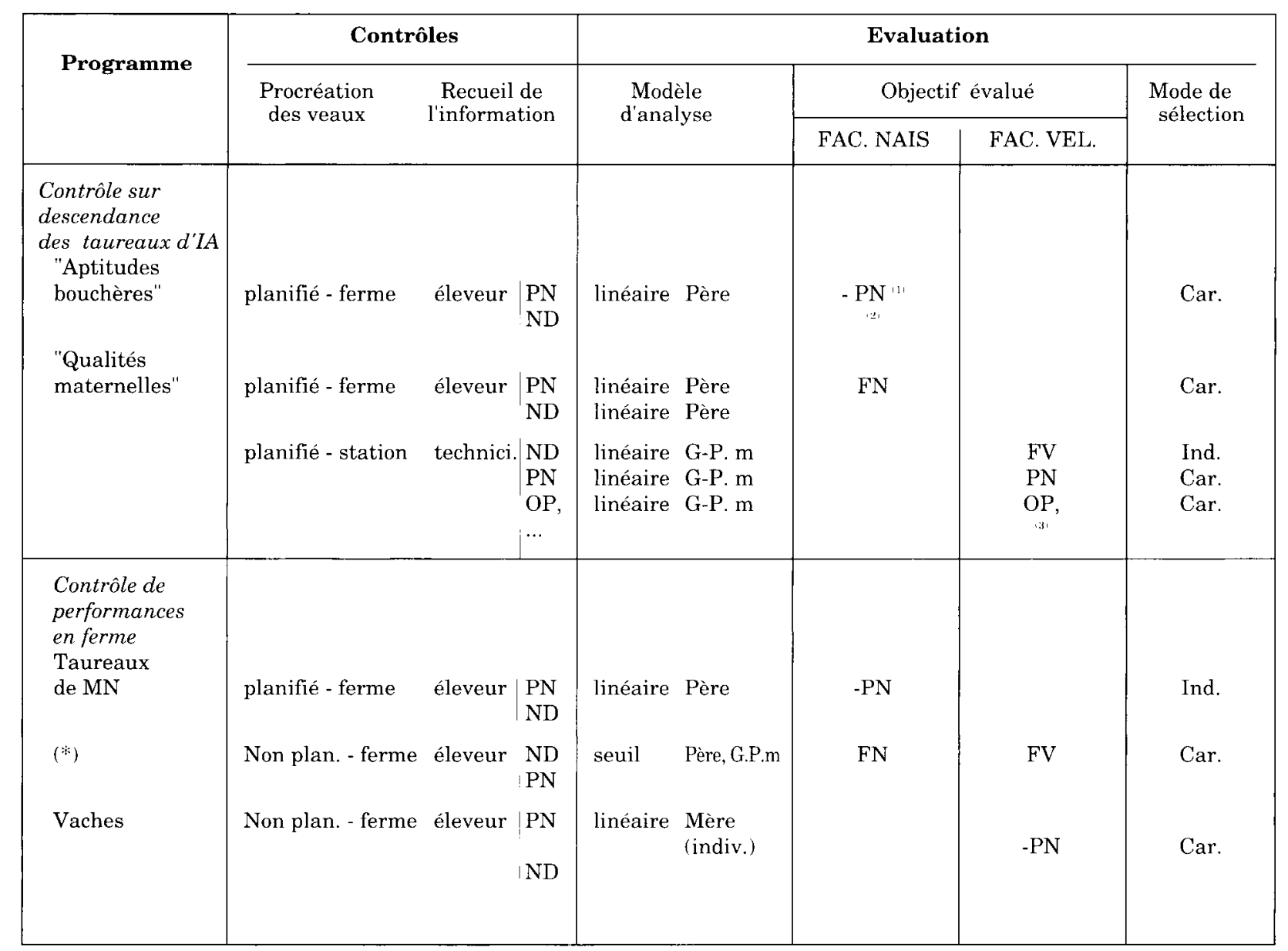

Tableau 4. Organisation du contrôle et de l'évaluation des facilités de naissance en races bovines à viande

(1) avec table de correspondance. (2) en projet, bivariate (PN-linéaire/ND-seuil) pour estimer FN

(3) en projet, multivariate (ND-seuil, $\mathrm{PN}$-linéaire, OP-linéaire,...) pour estimer F.V.
Car. : caractère indépendant.
Ind. : indice combiné.
ND : note de difficultés de naissance.
PN : Poids à la naissance.
OP : ouverture pelvienne.

FN ou FAC.NAIS. : "facilités de naissance" ou fréquence des naissances faciles (effet père).

FV ou FAC.VEL. : "facilités de vêlage" ou fréquence des vêlages faciles (effet mère ou grand-père maternel G-P. $m$ ). ${ }^{\star}$ ) Maine-Anjou 
- Le système de contrôle des performances des troupeaux allaitants sert aussi de support à l'évaluation des vaches et des taureaux. Pour les taureaux de monte naturelle ayant engendré des veaux dans des conditions définies (connexion entre élevages, ...), l'indice "facilités de naissance" (effet père) est obtenu à partir des poids à la naissance et il est inclus dans l'indice global servant à la qualification raciale de ces taureaux. Pour les vaches, leur indice "poids à la naissance" (effet mère) est utilisé pour apprécier leur aptitude maternelle au vêlage, notamment en tant que mères à taureaux. Une évaluation conjointe des effets pères et effets grands-pères maternels pour la fréquence des facilités de naissance avec un "modèle à seuil", représente une des étapes actuelles d'évolution de ces systèmes d'évaluation.

Quelle que soit l'importance des apports méthodologiques et zootechniques, la prise en compte des facilités de naissance dans l'objectif de sélection des bovins à viande reste une contrainte vis à vis de l'amélioration des aptitudes bouchères et des autres qualités maternelles.

\section{2 / Allaitement et croissance avant sevrage}

\section{1 / Importance}

L'environnement créé par la mère (allaitement, protection vis à vis des agressions, ...) a un rôle prépondérant dans les premiers stades de la vie du jeune ruminant. Il intervient non seulement sur la capacité de survie (vigueur, viabilité, ...) mais aussi sur la croissance du jeune jusqu'au sevrage. Le poids du jeune au sevrage conditionne une part notable de sa valeur commerciale pour l'engraissement (veaux broutards) ou pour l'abattage (agneaux et veaux de boucherie), et donc de la productivité du troupeaunaisseur ; ceci d'autant plus que le nombre de produits sevrés par mère et par cycle de reproduction est faible, comme c'est le cas pour les bovins. En tant que qualité maternelle, l'amélioration de la croissance du jeune de la naissance au sevrage à travers la maîtrise du couple "mère-jeunes", représente un des principaux objectifs de sélection des qualités d'élevage des ruminants à viande. Enfin, si dans certains systèmes de production d'ovins l'augmentation de la productivité passe par l'amélioration de la prolificité, il demeure indispensable de disposer de brebis capables d'élever ces portées multiples dans de bonnes conditions.

La croissance du jeune est sous la dépendance d'une part de sa propre capacité de croissance (capacité d'ingestion, efficacité alimentaire, ...) et, d'autre part, de son environnement maternel, au premier rang duquel prend place la production laitière de sa mère et le comportement maternel de celle-ci ou plus généralement l'établissement des relations comportementales mère-jeunes (figure 5). La quantité de lait consommée par le jeune intervient fréquemment pour 30 à $70 \%$ de la variabilité de sa croissance jusqu'au sevrage (poids ou gain de poids), comme le montrent les quelques exemples rapportés (tableau 5). L'éleveur-naisseur aura donc pour souci d'augmenter la production laitière de ses mères allaitantes - caractère qu'il sait sélectionnable chez les ruminants qui sont traits - et pour lequel il existe actuellement une variabilité importante entre races allaitantes (figure 6).
Figure 5. Déterminisme de la croissance avant sevrage.

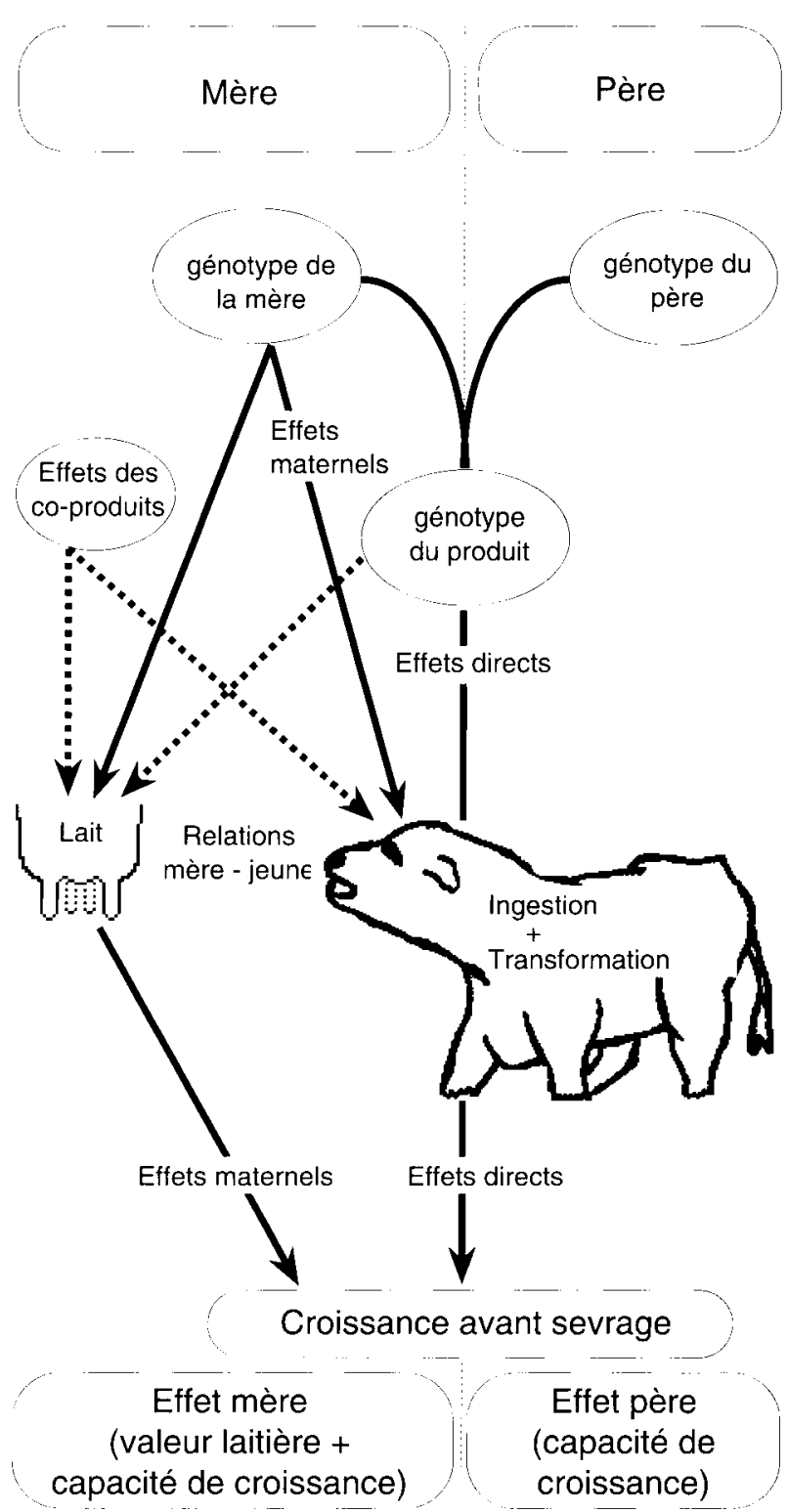

Figure 6. Production laitière de races bovines allaitantes et poids au sevrage de leurs veaux. (INRA - "Vachotron $1 "$ : Croisements entre races à viande).

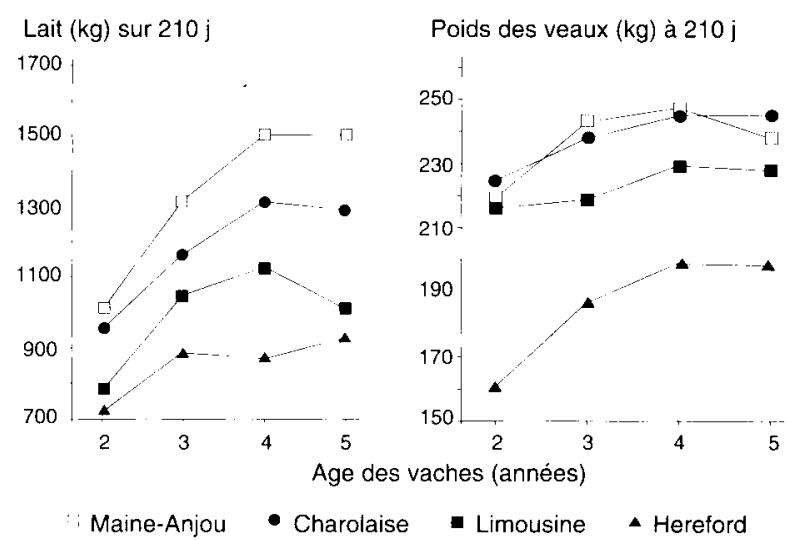


Tableau 5. Quelques exemples de corrélations phénotypiques entre poids ou croissance des jeunes et quantité de lait consommée.

Stade : mois (bovins) ou jours (ovins). Gi : corrélations avec la vitesse de croissance au stade i. Pi : corrélations avec le Poids vif au stade $i$. $T$ : croissance ou poids au sevrage.

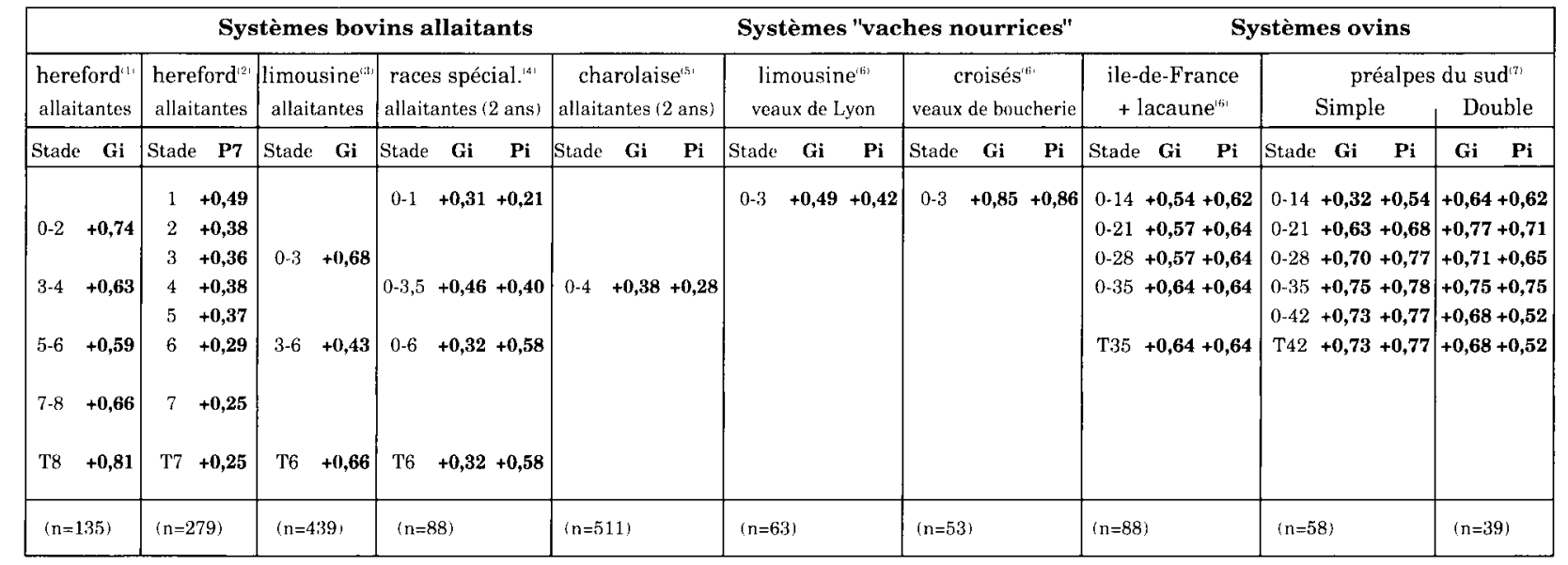

(1) Neuville 1962. (2) Rutledge et al 1971. Le Neindre et al 1976.

(4) INRA - "Vachotron 1" 1973 (non publié). (5) Ménissier 1975.

(6) Poujardieu 1969. (7) Ricordeau et Boccard 1961.

La lactation des mères allaitantes differe quelque peu des mères traites - (figure 7). Si la quantité totale de lait produit est moindre, la composition de celui-ci est plus riche. Le maximum de production est rapidement atteint mais la persistance de la production est élevée. Ceci est à relier à la stimulation importante par le jeune (plus de 6 tétées par jour en moyenne pour un jeune veau). D'ailleurs le nombre de jeunes allaités a une incidence directe sur la quantité de lait produite $(+20$ à $+60 \%$ pour des vaches allaitantes ayant adopté un veau supplémentaire). En outre la relation entre production laitière et croissance du jeune sera d'autant plus étroite que la quantité de lait disponible sera le facteur limitant la croissance et que le jeune n'aura pas d'alimentation complémentaire - ce qui est le cas durant la première période d'allaitement où le jeune ne rumine pas encore et dans les systèmes d'élevage extensifiés où la complémentation est trop onéreuse. Dans ces systèmes, le choix de la date de mise bas est déterminant pour faire coïncider l'accroissement de production laitière des mères avec celui des besoins et de la capacité d'ingestion des jeunes. Ainsi, l'objectif de sélection visera donc à accroître la capacité de croissance du ou des jeune(s) allaité(s) tout en améliorant la production de lait de la mère pour qu'elle ne soit pas le facteur limitant l'expression de cette capacité.

\section{2 / Déterminisme génétique}

La croissance jusqu'au sevrage est le type même de caractère dont le déterminisme relève d'un modèle génétique à effets directs et maternels (figure 5). Les "effets génétiques directs" représentent surtout la capacité de croissance du jeune, alors que les "effets génétiques maternels" (mesurés en terme de croissance du jeune, souvent appelés "valeur laitière" de la mère) englobent l'incidence de la production laitière maternelle et celle du comportement mère-jeune. Ainsi, comme pour les facilités de naissance, la croissance du jeune allaité ne recouvre pas la même siginification biologique selon qu'il s'agit des variations entre pères (effet père) ne concernant que des effets génétiques directs sur la croissance du jeune, ou des variations entre mères ou grands-pères maternels (effet mère, désigné par "aptitude à l'allaitement") prenant en compte les effets génétiques directs et les effets génétiques résultant de l'incidence des caractères maternels. Le critère d'évaluation des reproducteurs ne sera donc pas nécessairement le même pour

Figure 7. Courbe de lactation de vaches limousines (d'après Le Neindre et al 1976).

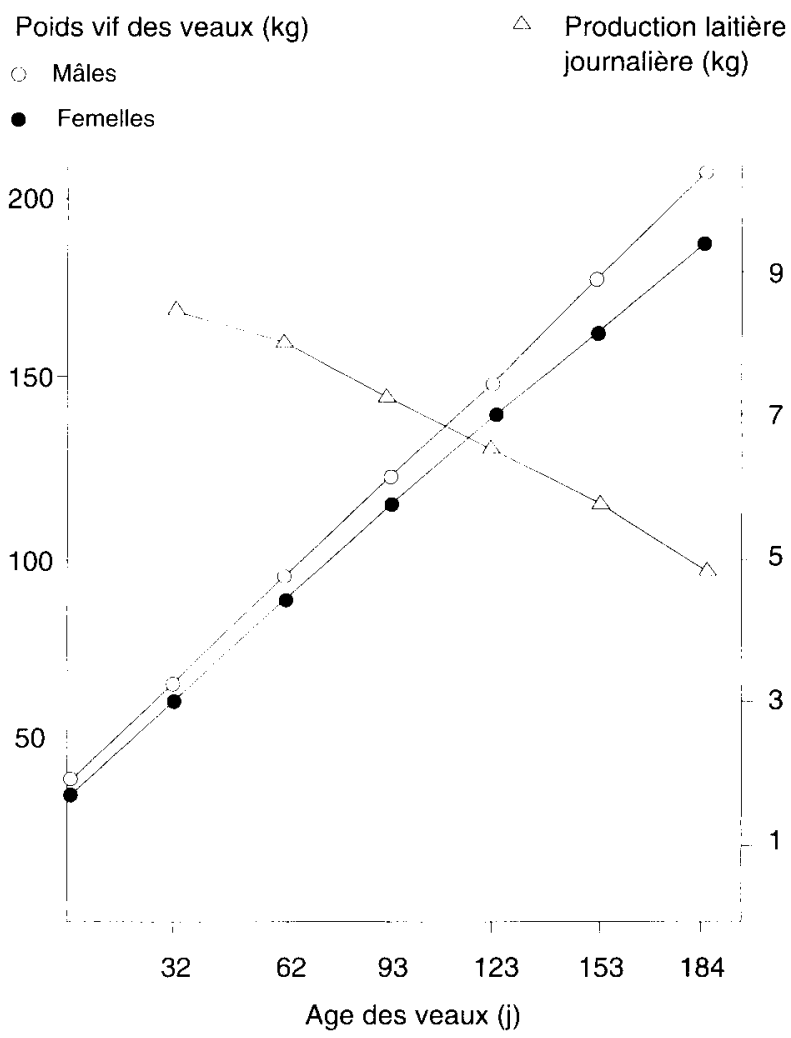

(439 lactations, contrôle mensuel, $1470 \mathrm{~kg}$ pour 180 j) 
sélectionner uniquement la croissance du jeune comme composante de ses aptitudes bouchères ou pour améliorer l'aptitude à l'allaitement ou la valeur laitière des mères.

Les principaux paramètres génétiques relatifs à l'allaitement (tableau 6) laissent présager des bonnes possibilités de sélection de la croissance avant sevrage puisque les héritabilités des effets mères ou des effets maternels sont suffisamment élevées tout comme pour les effets directs (Poivey et al 1987, Ménissier et Frisch 1992). Toutefois, le point dominant est l'existence d'une relation génétique négative entre effets directs et effets maternels, conduisant à des corrélations plutot faibles entre effets pères et effets mères (ou grands-pères maternels). Cette relation entre effets directs et maternels nous semble d'autant plus négative que les systèmes d'élevage sont plus extensifs, c'est à dire que la production laitière des mères est limitée au regard des besoins du jeune et ceci surtout en allaitement multiple. Par ailleurs pour les bovins allaitants, il a été émis qu'une part de cet antagonisme peut être due à des phénomènes environnementaux maternels (Ménissier 1975) : les génisses ayant eu une croissance élevée en raison de la production laitière importante de leur mère, auraient un moins bon développement du tissu mammaire et de ce fait produiraient des veaux plus légers au sevrage. Signalons ici que les rares estimations en bovins allaitants (primipares Charolaises en station) indiquent que la production laitière est héritable $\left(\mathrm{h}^{2}=0,4\right)$ et très liée à l'aptitude à l'allaitement ( $\mathrm{rg}=+0,8$ ) (Ménissier 1975).

Outre la sélection des effets directs sur la croissance avant sevrage, une sélection spécifique sur les effets mères ou grands-pères maternels est nécessaire pour améliorer également la valeur laitière ou l'aptitude à l'allaitement. Ceci est d'autant plus important à considérer que, comme pour les facilités de naissance, la sélection de la croissance post-natale avant sevrage n'est probablement pas indépendante de la taille ou format des mères et de la précocité de développement (maturité à la naissance, au sevrage, à l'abattage, à la mise en reproduction, ...) donc des caractères maternels comme la capacité d'ingestion et d' "épargne alimentaire" ou les composantes des aptitudes bouchères. Ces implications pourraient différer entre ovins et bovins à viande en raison notamment de l'importance de la prolificité ou des différences de maturité lors de l'engraissement .

\section{3 / Critères et contrôles de performances}

L'aptitude à l'allaitement ou la valeur laitière peut être appréciée à partir de différents critères : soit par mesure directe de la production laitière lors de l'allaitement, soit par la croissance des jeunes d'une mère à un stade où le gain de poids reflète le mieux la quantité de lait disponible, ingérée et transformée par les produits.

Les difficultés de mesure directe de la production laitière sont nombreuses. Le comportement maternel vis à vis d'une traite occasionnelle correspond à une situation de stress (contention, injection d'ocytocine pour déclencher l'éjection du lait, ...) et ne correspond pas nécessairement aux quantités dont disposent le ou les jeunes lors des multiples tétées. Cette quantité journalière de lait est cependant estimable par pesées avant et après tétées selon un protocole adapté (séparation des mères et des jeunes, tétées surveillées à intervalles réguliers). 3 contrôles journaliers lors de la lactation procurent une bonne estimation de la quantité fournie lors de la lactation $(r=0,8$ à 0,9$)$. C'est ce qui se pratique en troupeau expérimental ( 3 contrôles) et en station de contrôle des qualités maternelles ( 2 contrôles) chez les bovins. Ce type de mesure est difficilement transposable dans les élevages en contrôle de performances.

En revanche la mesure de la croissance du ou des jeunes considérée comme aptitude de la mère (effet mère), au moment où cette croissance est la plus corrélée à la production laitière ou à la quantité de lait bue (tableau 5) (Ricordeau et Boccard 1961), est la solution qui a été adoptée pour les contrôles de performances dans les élevages. Même s'il existe des stades

Tableau 6.
Paramètres
génétiques de
la croissance
avant sevrage
des bovins et
ovins
allaitants.

\begin{tabular}{|c|c|c|c|c|c|c|}
\hline & \multicolumn{3}{|c|}{ Bovins ${ }^{1 !}$} & \multicolumn{3}{|c|}{ Ovins ${ }^{i 21}$} \\
\hline & $\begin{array}{l}\text { Poids au } \\
\text { sevrage }\end{array}$ & $\begin{array}{l}\text { Croissance } \\
\text { nais.-sevra. }\end{array}$ & $\begin{array}{l}\text { Poids à la } \\
\text { naissance }\end{array}$ & $\begin{array}{l}\text { Poids à } \\
10 \text { jours }\end{array}$ & $\begin{array}{l}\text { Gain de } \\
10-30 \text { jours }\end{array}$ & $\begin{array}{l}\text { Poids à } \\
30 \text { jours }\end{array}$ \\
\hline $\mathbf{h}^{2}$ direct ou père & 0,15 à 0,40 & 0,15 à 0,20 & 0,10 à 0,40 & 0,37 & 0,42 & 0,41 \\
\hline $\mathbf{h}^{2}$ maternel & 0,15 à 0,35 & 0,15 à 0,35 & 0,05 à 0,25 & 0,30 & 0,29 & 0,33 \\
\hline $\mathbf{h}^{2}$ mère $^{\text {ial }}$ & 0,10 à 0,30 & 0,10 à 0,25 & 0,10 à 0,40 & 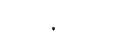 & . & . \\
\hline $\mathbf{r}_{\mathbf{g}}$ (père/mère) & $+0,1 \grave{a}+0,4$ & $+0,1 \grave{a}+0,4$ & 0 à $+0,5$ & . & . & . \\
\hline $\mathbf{r}_{\mathbf{g}}($ direct/maternel $)$ & $-0,4$ à 0 & $-0,5$ à 0 & $-0,4$ à 0 & $-0,62$ & $-0,70$ & $-0,61$ \\
\hline \multicolumn{7}{|c|}{$\mathbf{r}_{\mathbf{g}}($ direct/caractère maternel $)$} \\
\hline Format/taille/poids & \multirow{2}{*}{\multicolumn{2}{|c|}{$\begin{array}{c}0 \grave{a ̀ ~}+ \\
+0,4 \text { à }+0,8\end{array}$}} & $+0,4 \grave{a}+0,6$ & & 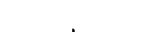 & . \\
\hline Production laitière & & & & $(+)$ & $(+)$ & . \\
\hline Précocité/maturité & \multicolumn{2}{|c|}{. } & - à 0 & . & . & . \\
\hline Muscularité & \multicolumn{2}{|c|}{. } & $0 \grave{a ̀}+$ & . & . & . \\
\hline \multicolumn{7}{|c|}{$r_{\mathrm{g}}$ (mère/aptitudes bouchères) } \\
\hline Croissance & \multicolumn{2}{|c|}{$(-$ à 0$)$} & $+0,3$ à $+0,6$ & $(+)$ & $(+)$ & \\
\hline Poids total viande & \multicolumn{2}{|c|}{$(-\grave{a} 0)$} & $+0,2 \grave{a}+0,4$ & $(+)$ & $(+)$ & . \\
\hline Croissance musculaire & \multicolumn{2}{|c|}{$(?)$} & $(+)$ & . & . & . \\
\hline
\end{tabular}

(1) Valeurs les plus probables .() Valeurs incertaines. (2) Ile-de-France Poivey et al 1987.

(a) ou grand-père maternel. 
optima quelque peu différents selon les systèmes et mode d'élevage (allaitement simple ou multiple, complémentation ou non et plus ou moins précoce, ...), la vitesse de croissance entre les âges de 10 et 30 jours chez les ovins et le poids à l'âge de 120 jours chez les bovins, obtenus à partir de 2 pesées spécifiques, sont les critères de croissance retenus dans les protocoles nationaux respectifs de contrôle et évaluation de l'aptitude à l'allaitement. La période périnatale a été exclue de l'élaboration de ces critères en raison de sa forte dépendance envers des phénomènes autres que l'allaitement (difficultés de naissance, établissement des relations mère-jeunes, ....). Ces contrôles s'insèrent dans le système général de contrôle des performances des troupeaux allaitants et notamment celui de pesées des jeunes avant sevrage (bovins et ovins) ou abattage (ovins), réalisé lors de passages systématiques d'un technicien spécialisé (chaque 3 semaines ou 3 mois, respectivement pour les ovins ou bovins). Les naissances étant étalées sur des périodes plus ou moins longues, les pesées ont donc lieu à des âges variables et un jeune peut être pesé 3 (bovins) ou 4 (ovins) fois ; les 2 premières pesées sont utilisées pour le contrôle d'aptitude à l'allaitement et les 2 dernières pour estimer la capacité de croissance du jeune (vitesse de croissance de 30 à 70 jours ou poids à 210 jours pour respectivement les ovins et bovins) et évaluer sa valeur individuelle ou celle de son père (effets directs). Face aux coûts et contraintes de ce système de pesées en troupeaux allaitants, il est fréquemment demandé de simplifier ce dispositif notamment en réduisant le nombre de pesées par individu. L'étude réalisée chez les ovins (tableau 7) (Poivey et al 1987) montre qu'en fait seule la suppression de la 3ème pesée induirait une faible perte de précision sur la mesure de la croissance individuelle, mais tout en obligeant à répertorier les agneaux à ne pas peser lors de chaque passage sans pour autant réduire le nombre de passages dans l'élevage.

\section{4 / Evaluation et sélection des reproducteurs}

L'évaluation génétique de l'aptitude à l'allaitement se pratique de manière identique chez les ovins et bovins, avec quelques spécificités de réalisation compte tenu des particularités biologiques et du contexte d'exploitation et sélection de chaque espèce (Ménissier 1988).

\section{a / Contrôles en station (taureaux d'IA)}

L'aptitude à l'allaitement est une des qualités maternelles des taureaux d' IA (effets grands-pères maternels) évaluées sur leurs filles en station conjointement aux facilités de vêlage vues précédemment. Une valeur génétique laitière et une valeur génétique maternelle pour la croissance avant sevrage sont estimées respectivement à partir du contrôle de la production laitière des filles et de la croissance de leurs veaux jusqu'à 4 mois. L'indice "allaitement" qui combine essentiellement ces deux valeurs génétiques, représente l'aptitude à produire des veaux plus lourds au sevrage, qu'auront les filles procréées par ces taureaux. Cet indice entre pour 20 à $30 \%$ dans l'indice global servant pour l'agrément "qualités maternelles" des taureaux ; ce qui se traduit en pratique par une différentielle de sélection notable (tableau 8).

\section{b / Contrôles en ferme (ovins et bovins)}

Jusqu'à présent l'évaluation génétique de l'allaitement repose sur l'analyse de la croissance 10-30 jours (ovins) ou du poids à 120 jours (bovins) des jeunes, après prise en compte des multiples facteurs ayant une incidence sur cette croissance (année, troupeau, saison, parité, sexe, mode de naissance, conduite d'élevage, ...) et standardisation de la variabilité. La croissance des jeunes est alors considérée comme une performance individuelle répétée de la mère (effet mère). Un indice "allaitement" pour les vaches ayant eu au moins 3 produits ou un indice "valeur laitière" pour les brebis est fourni aux éleveurs pour les aider au choix des mères de renouvellement. Par exemple, les qualifications raciales des vaches allaitantes reposent pour une large part sur cet indice (vaches qualifiées : vaches dont l'indice est supérieur à la moyenne ou de $+1 / 2$ à $3 / 4$ d'écart-type selon la qualification). Chez les ovins un indice sur descendance est également élaboré pour les béliers (pères des brebis).

Une évolution importante est en cours grâce au recours à un modèle génétique d'évaluation mieux adapté au déterminisme du caractère (modèle à effets directs et maternels) et à ce type de contrôles des performances d'individus apparentés et sélectionnés (modèle individu). Ce nouveau système permet d'obtenir une estimation conjointe des effets génétiques directs et maternels pour chaque individu (produit ou parents). Il conviendra alors de combiner ces deux estimations (la "valeur laitière" ou les effets génétiques maternels, avec la "croissance" ou les effets génétiques directs) en un indice de "valeur allaitante" traduisant l'objectif racial de sélection pour une amélioration relative plus ou moins grande de la production laitière vis à vis de la capacité de croissance du ou des jeunes. La mise en oeuvre de tel système est toutefois conditionnée à la disposition,

\begin{tabular}{|c|c|c|c|c|c|c|c|c|}
\hline \multirow{2}{*}{$\begin{array}{l}\begin{array}{l}\text { Suppression } \\
\text { de la pesée }\end{array} \\
\text { P1 }\end{array}$} & \multicolumn{2}{|c|}{$\begin{array}{c}\text { Poids } \\
30 \mathrm{j}(\mathrm{kg})\end{array}$} & \multicolumn{2}{|c|}{$\begin{array}{c}\text { Poids } \\
70 \mathrm{j}(\mathrm{kg})\end{array}$} & \multicolumn{2}{|c|}{$\begin{array}{l}\text { Croissance } \\
10-30 \mathrm{j}(\mathrm{g} / \mathrm{j})\end{array}$} & \multicolumn{2}{|c|}{$\begin{array}{l}\text { Croissance } \\
30-70 \mathrm{j}(\mathrm{g} / \mathrm{j})\end{array}$} \\
\hline & $-2 \pm 6$ & $(7 \%)$ & - & - & $38 \pm 95$ & $(64 \%)$ & $4 \pm 16$ & $(5 \%)$ \\
\hline P2 & $+3 \pm 9$ & $(15 \%)$ & - & - & $-19 \pm 47$ & $(31 \%)$ & $-8 \pm 23$ & $(10 \%)$ \\
\hline P3 & - & & $0 \pm 10$ & $(6 \%)$ & - & - & $-1 \pm 26$ & $(12 \%)$ \\
\hline $\mathrm{P} 4$ & - & & $-5 \pm 21$ & $(21 \%)$ & & - & $-14 \pm 52$ & $(35 \%)$ \\
\hline P1, P3 & $-1 \pm 6$ & $(6 \%)$ & $0 \pm 10$ & $(6 \%)$ & $51 \pm 81$ & $(55 \%)$ & $4 \pm 29$ & $(15 \%)$ \\
\hline $\mathrm{P} 2, \mathrm{P} 4$ & $3 \pm 9$ & $(15 \%)$ & $-8 \pm 19$ & $(17 \%)$ & $19 \pm 47$ & $(31 \%)$ & $-28 \pm 51$ & $(35 \%)$ \\
\hline
\end{tabular}

(1) 4 pesées obtenues entre l'âge de 1 à 84 jours, lors de 4 tournées (chaque 3 semaines). () perte de précision (variance résiduelle du biais/variance de la variable, en \%).

Source : Poivey et al 1987 - agneaux lle-de-France (1972 à 1985).

Tableau 7. Allègement du protocole de contrôle de croissance des agneaux en ferme. Analyse des écarts individuels (moyenne, écart-type résiduel et perte de précision). 
Tableau 8. Sélection réalisée sur descendance en station pour les qualités maternelles des taureaux d'insémination artificielle.

\begin{tabular}{|c|c|c|c|}
\hline $\begin{array}{l}\text { Race } \\
\text { Station } \\
\text { Début } \\
\text { Nombre taureaux/an }\end{array}$ & $\begin{array}{c}\text { Charolaise } \\
\text { Agonges (03) } \\
1968-70 \\
15\end{array}$ & $\begin{array}{c}\text { Limousine } \\
\text { Uzerche (19) } \\
\text { 1972-74 } \\
6\end{array}$ & $\begin{array}{c}\text { Blonde d'Aquitaine } \\
\text { Casteljaloux (47) } \\
1972-74 \\
6\end{array}$ \\
\hline Bilan depuis (nbre séries) & $1975-77(14)$ & $1980-82(6)$ & $1976-78(11)$ \\
\hline Nombre taureaux évalués & 147 & 54 & 67 \\
\hline \% agréés "qualités maternelles" & $31 \%$ & $33 \%$ & $30 \%$ \\
\hline \multicolumn{4}{|l|}{ Valeurs génétiques des agréés } \\
\hline - Poids à 18 mois (kg) & +11 & +8 & +10 \\
\hline - Développement musculaire (pt) & $+0,2$ & $+1,4$ & $+0,8$ \\
\hline - Développement squelettique (pt) & $+2,5$ & $+0,8$ & $+1,9$ \\
\hline * Indice de croissance ${ }^{(a)}$ & 110 & 112 & 109 \\
\hline - Précocité sexuelle (\%) & $+1,7$ & $+5,5$ & $+1,6$ \\
\hline - Fécondation (\%) & $+2,6$ & $+3,1$ & $+4,9$ \\
\hline * Indice de fertilité $(\mathrm{a})$ & 103 & 104 & 104 \\
\hline - Vêlages faciles (\%) & $+5,7$ & $+0,2$ & $+5,3$ \\
\hline - Poids à la naissance des veaux (kg) & $+0,1$ & $+0,2$ & $-0,1$ \\
\hline - Ouverture pelvienne (cm2) & +3 & +0 & +3 \\
\hline - Poids au vêlage (kg) & +15 & +9 & +11 \\
\hline * Indice de facilité de vêlage ${ }^{(a)}$ & 107 & 100 & 107 \\
\hline - Lait à 4 mois $(\mathrm{kg} / \mathrm{j})$ & $+0,2$ & $+0,3$ & $+0,3$ \\
\hline - Poids des veaux à 4 mois $(\mathrm{kg})$ & $+1,5$ & $+2,3$ & $+2,9$ \\
\hline à 6 mois $(\mathrm{kg})$ & $+1,6$ & $+2,3$ & $+1,7$ \\
\hline * Indice d'allaitement ${ }^{(a)}$ & 105 & 109 & 109 \\
\hline * Indice "qualités maternelles" (b) & 107 & 106 & 108 \\
\hline
\end{tabular}

(a) Valeur standardisée (100=moyenne de série, 20=1 écart-type génétique).

(b) combinaison des 4 indices.

outre d'un système informatique performant, d'une information généalogique suffisante (paternité connue pour les agneaux ou les bovins accouplés en estives, ...), d'un contrôle de performances suffisamment descriptif vis à vis des sources de variation individuelle de la croissance (état physiologique des mères, groupes de conduite, traitements préférentiels, ...) et de bonnes estimations des paramètres génétiques relatifs aux différentes populations et systèmes de conduites (ce qui n'est pas le cas notamment pour les effets maternels et leurs corrélations avec les effets directs). Pour les ovins, le modèle animal (ou individu) de description des données pose des problèmes spécifiques en raison de la prolificité, puisque la production de lait d'une brebis est partagée entre plusieurs jeunes (individus) et le nombre et les caractéristiques des co-jumeaux (triplets) allaités ont une incidence sur l'expression de sa production laitière. La seule croissance d'un agneau n'a pas la même valeur informative pour les effets génétiques maternels ("valeur laitière") selon que, par exemple, son cojumeau ait été allaité ou non avec lui. Il devient alors indispensable dans ce cas d'utiliser la viabilité des agneaux comme une autre information prédictrice de la valeur génétique maternelle pour la croissance avant sevrage (modèle multivariable).

\section{Conclusion}

De l'analyse de ces deux exemples de qualités d'élevage ou qualités maternelles des ruminants allaitants, on peut tirer les enseignements généraux suivants vis à vis de leur sélection :

- Les qualités maternelles impliquent des relations inévitablement complexes entre la mère et son ou ses jeunes, de leur conception à leur sevrage ; ce qui oblige à une modélisation simplifiée mais cohérente des phénomènes biologiques mis en cause et de leur régulation.

- La prise en compte des qualités maternelles dans la sélection ne peut se faire sans considérer les répercussions qu'elles induisent sur les autres aptitudes, notamment au travers des modifications provoquées sur les caractéristiques biologiques prédominantes et des antagonismes qui pourraient en résulter.

- L'efficacité de la mise en oeuvre d'une sélection des qualités maternelles est conditionnée par la mobilisation simultanée de compétences dans les domaines cognitifs et appliqués relatifs à la biologie des ruminants et aux méthodologies de sélection. 


\section{Références bibliographiques}

Foulley J.L., Ménissier F., 1978. Sélection de l'aptitude au vêlage en race à viande françaises. In: La sélection de l'aptitude au vêlage, Bull. tech. Dép. génét. anim. (INRA), $\mathrm{n}^{\circ} 27,55-77$.

Giraudeau L., 1990. Etude de la variabilité génétique de la race Parthenaise. Mémoire fin études, INA-Paris-Grignon, $127 \mathrm{p}$.

ITEB (Institut Technique de l'Elevage Bovin), 1991. Chap.1 - Fonctionnement. technico-économique de l'exploitation. Chap.2 - Le Troupeau : génétique, reproduction et santé. In: Troupeau allaitant - mode d'emploi : raisonner pour mieux conduire, Grenet N. (éd). p.9-26, p.27-121, Technipel, Paris, France.

Le Neindre P., Petit M., Tomassone R., Roux C., 1976. Production laitière des vaches allaitantes et croissance de leurs veaux. I - Race Limousine. Ann. Zootech., 25, 221-224.

Manfredi E., 1990. Analyse génétique des conditions de naissance chez les bovins par le modèle à seuils. Thèse Doct., Univ. Paris-Sud Orsay, 245p.

Manfredi E., Ducrocq V., Foulley J.L., 1990. Genetic analysis of dystocia in dairy cattle. J. Dairy Sci., 74, 17151723

Ménissier F., 1975. Comments on optimization of cattle breeding schemes : beef breeds for suckling herds : a review. Ann. Génét. Sél. anim., 8, 71-87.

Ménissier F., 1979. Difficultés de mise bas chez les bovins. In: Action conjointe des effets directs et maternels des gènes sur les caractères de production. Bull. tech. Dép. génét. anim. (INRA), n²9-39, 132-190.

Ménissier F., 1988. La sélection des races bovines à viande spécialisées en France. Compte-rendus du 3ème Congrès mondial de reproduction et sélection des ovins et bovins à viande, Paris-La Villette, 19-23 juin 1988. Vol.2, 215-236. INRA, Paris, France.

Ménissier F., Foulley J.L., 1978. Situation actuelle des problèmes de vêlage dans la Communauté économique européenne : importance des difficultés de vêlage et de la mortinatalité chez les bovins à viande. In: La sélection de l'aptitude au vêlage. Bull. tech. Dép. génét. anim. (INRA), $\mathrm{n}^{\circ} 27,5-54$.

Ménissier F., Foulley J.L., 1979. Present situation of calving problems in the E.E.C. : incidence of calving difficulties and early calf mortality in beef herds. In: Calving problems and early viability of the calf. Hoffmann B., Mason I.L., Schmidt J. (eds). 30-85. Martinus Nijhoff Publishers, The Hague, The Netherlands.

Ménissier F., Frisch J.E., 1992. chap.3 - Genetic improvement of beef cows. In: Beef cattle production, Jarrige R. et Béranger C. (eds). (World Animal Science. C, Production-system approach ; 5), p55-85 Elsevier Sciences Publisher, Amsterdam, The Netherlands.

Ménissier F., Petit M., 1984. Poids et vitalité des veaux à la naissance : leurs implications zootechniques. In: Physiologie et pathologie périnatales chez les animaux de ferme, Jarrige R. (éd.). 279-308, INRA, Paris, France..

Neville (Jr) W.E., 1962. Influence of dam's milk production and other factors on 120-and 240-day weight of Hereford calves. J. Anim. Sci., 21, 315-320.

Poivey J.P., Niare T., Cournut J., Bibé B., 1987. Etude des possibilités d'allègement du protocole de pesée des agneaux dans les élevages français. 38ème Réun. ann. F.E.Z., 28 septembre - ler octobre 1987, Lisbonne (Portugal). Vol.II, 1021 (abstr.), 11 pages.

Poujardieu B., 1969. Recherche d'une méthode d'estimation de la production laitière des femelles ovines et bovines pendant la phase d'allaitement. Ann. Zootech., 18, 299-315.

Ricordeau G., Boccard R., 1961. Relation entre la quantité de lait consommée par les agneaux et leur croissance. Ann. Zootech., 10, 113-125.

Rutledge J.J., Robison O.W., Ahlschwede W.J., Legates J.E., 1971. Milk yield and its influence on 205-day weight of beef calves. J. Anim. Sci., 33, 563-567. 